hep-th/9912025

IFP-9912-UNC

HUB-EP-99/62

\title{
Horizontal and Vertical Five-Branes in Heterotic/F-Theory Duality
}

\author{
Björn Andreast国 and Gottfried Curio*2 \\ $\dagger$ Department of Physics and Astronomy \\ University of North Carolina, Chapel Hill, NC 27599-3255, USA \\ *Humboldt-Universität zu Berlin, \\ Institut für Physik, D-10115 Berlin, Germany
}

\begin{abstract}
We consider the heterotic string on an elliptic Calabi-Yau three-fold with five-branes wrapping curves in the base ('horizontal' curves) of the Calabi-Yau as well as some elliptic fibers ('vertical' curves). We show that in this generalized set-up, where the association of the heterotic side with the $F$-theory side is changed relative to the purely vertical situation, the number of five-branes wrapping the elliptic fibers still matches the corresponding number of $F$-theory three-branes.
\end{abstract}

1 bandreas@physics.unc.edu, supported by U.S. DOE grant DE-FG05-85ER40219

2 curio@physik.hu-berlin.de 


\section{Introduction}

The compactification of the heterotic string on a Calabi-Yau three-fold with a vector bundle over it, which breaks part of the $E_{8} \times E_{8}$ gauge symmetry, leads to $N=1$ supersymmetric vacua in four dimensions.

In particular the class of elliptically fibered Calabi-Yau three-folds $Z$ has the double advantage to admit a direct construction of the vector bundles and to allow a dual description in terms of $F$-theory compactified on elliptically fibered Calabi-Yau four-folds $X$ [1].

To obtain a consistent heterotic compactification on elliptic Calabi-Yau three-folds, the anomaly cancellation condition requires to include a number $a_{f}$ of five-branes wrapping the elliptic fibers. It has been shown that these five-branes match precisely the number $n_{3}$ of space-time filling three-branes necessary for tadpole cancellation on the $F$-theory side [1], [2] and so giving a heterotic string explanation of the number of three-branes.

Here we will be interested in a slightly more general situation, where one admits also five-branes wrapping holomorphic curves in the base $B_{2}$ of the elliptic Calabi-Yau threefold (we will take $B_{2}$ to be a Hirzebruch surface $F_{n}$ of $n=0,1,2$ or a del Pezzo surface $d P_{k}, k=0, \ldots, 8$; the case of an Enriques surface could be treated along similar lines). The picture which emerges is, the five-branes wrapping the elliptic fibers map to the $F$ theory three-branes whereas a five-brane wrapping a curve in the base $B_{2}$ of the elliptic Calabi-Yau three-fold $Z$ corresponds to a blow-up of the base $B_{3}$ along the curve in the common $B_{2}$ of the elliptic Calabi-Yau four-fold $X$ on the $F$-theory side (recall that $B_{3}$ resp. $X^{4}$ is a $P^{1}$ resp. $K 3$ fibration over $B_{2}$ ).

Several aspects of this generalized set-up were discussed in [3], [4], [5], mainly in the case of wrapping curves of genus zero in the base and in the extreme situation when the bundles have trivial structure group leading to an unbroken $E_{8} \times E_{8}$ gauge group. The possibility of wrapping five-branes on curves in the base (or even in $Z$ ) was also carefully

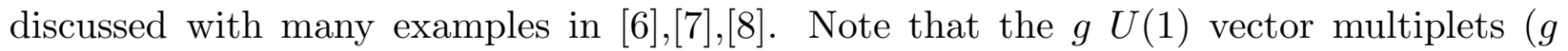
being the genus of $C$ ) coming from reduction of the two-form of self-dual field-strength on the five-brane, are associated with corresponding modes from three-cycles in the blown-up base $\tilde{B}_{3}$ and the four-form of self-dual field-strength of type IIB on the $F$-theory side.

Note that a curve lying in the base $B$ will have in general an interesting set of deformations which will play a role below. In the set-up with five-branes wrapping only fibers the brane-parameters on both sides took care of themselves. Remember that the 'three 
coordinates' of such a three-brane, considered as a point in the $\mathbf{P}^{1}$ bundle $B_{3}$ over the visible $B_{2}$, are simply given by the base-point of the corresponding elliptic fiber on the heterotic side which is trivially given on the common $B_{2}$ and the 'third coordinate', i.e. the fact that there is still a moduli space $\mathbf{P}^{1}$ in the game, is given by a complex scalar whose real resp. imaginary part is given by the position in the $M$-theory interval $S^{1} / Z_{2}$ in the eleventh dimension resp. the axion $a$ corresponding to the reduction of the self-dual three-form on the five-brane (note that this $S^{1}$ must collapse to a point at the boundaries of the interval - thus leading to the third, missing complex coordinate, parametrizing on the $F$-theory side the $P^{1}$ fiber over the common $B_{2}$ - as there one has a transition to $E_{8}$ instantons without the interval position modulus and so without $a$ too (the five-brane three-form being odd it must vanish at the orbifold fixed planes anyway); cf. [5], [8]). Note also that the heterotic string on a wrapped $T^{2}$ is equivalent to the $S O(32)$ heterotic string and a small instanton-fivebrane has a non-perturbative $S U(2)$ which has as moduli space on $T^{2}$ exactly a $\mathbf{P}^{1}$ (in general an $S U(n)$ bundle on $T^{2}$ has as moduli space a $\mathbf{P}^{n-1}$ ).

Of course the expectation is still that the five-branes wrapping elliptic fibers correspond to the $F$-theory three-branes and this should not be changed by the occurrence of the 'other', horizontal five-branes. However the actual proof of the matching in the purely vertical case [1] did not associate these objects directly with each other (although of course this is the obvious underlying intuition) but rather computed the number of the relevant five-branes resp. three-branes independently on both sides in data of the common base $B_{2}$. To get the matching one had to use then an association of both sides (this was the relation $\eta_{1,2}=6 c_{1}\left(B_{2}\right) \pm t$, respecting the condition $\eta_{1}+\eta_{2}=12 c_{1}\left(B_{2}\right)$, where $t$ characterizes the $P^{1}$ fibration of $B_{3}$ over $B_{2}$ ). But we will have a non-trivial horizontal five-brane (of class $\left.W_{B}\right)$ and so the mentioned condition changes to $\eta_{1}+\eta_{2}+W_{B}=12 c_{1}\left(B_{2}\right)$ on the one hand and $B_{3}$ is modified by the blow-up on the other hand. This makes the numerical matching non-trivial.

In order to proof the matching of vertical five-branes and three-branes in this generalized set-up we will facilitate the analysis by considering a five-brane wrapping a smooth irreducible curve $C$ representing a class $W_{B}$ and thus blow up once the $F$-theory base $B_{3}$. We will separate our analysis into parts starting with the simplest case of a heterotic string compactification on $Z$ with an $E_{8} \times E_{8}$ vector bundle corresponding under duality to $F$-theory on a smooth $X$ (where smooth means that the elliptic fibers of $X$ degenerate in codimension one not worse than $I_{1}$ in the Kodaira classification). After explaining how a blow-up of $W_{B}$ in the base $B_{3}$ of $X$ changes the Euler number of $X$ 
and therefore the number of three-branes, we follow the strategy of [1] and express the number of three-branes and five-branes in comparable data on the common base $B_{2}$. Then we proceed to models with an $A-D-E$ gauge group and consider a heterotic model with an $E_{8} \times V_{2}$ vector bundle where $V_{2}$ can be $E_{7}, E_{6}, S U(n)$ giving corresponding unbroken gauge groups. The dual $F$-theory model (assuming no further monodromy effects, which could alter the gauge group, being present there) is described by compactifying on an $X$ whose elliptic fibers degenerate of type $A-D-E$. For simplicity we will admit only that the fibers degenerate over codimension one in $B_{3}$ (which can be done by adjusting the $\mathbf{P}^{\mathbf{1}}$ bundle over $B_{2}$ respectively the $\eta$ class on the heterotic side). In order to 'fill out' $V_{2}$ with enough instanton number (measured by the $\eta$-bound) we have to perform the $W_{B^{-}}$shift in the $E_{8}$ bundle. One has, just as in the smooth case, again an explicit formula for the Euler number, which was recently proved in [9]. However, as this ansatz is not immediately adapted to the blow-up procedure which destroys the usual $P^{1}$ fibration structure of $B_{3}$, we will proceed in the proof of the five-brane/three-brane correspondence more easily by adopting the strategy of [2]. In the latter one expresses the number of three-branes again via the Euler number, which this time is then in turn expressed by the Hodge numbers, they again by the heterotic data (including the index of bundle moduli 'inside' the complex structure deformations of $X$ ); then the close similarity of the expression for the index of bundle moduli with the expression for the second Chern class of the vector bundle is used to transport directly the number of five-branes to the $F$-theory side. We will reduce our more general situation $\left(W_{B} \neq 0\right)$ to this original case $\left(W_{B}=0\right)$ by collecting the changes in the Hodge numbers of the fourfold induced by the blow-up procedure resp. by the 'change' in heterotic bundle moduli (in a sense made precise below) when transported to the $F$-theory side; taken together with the deformations of the chosen curve $C$ these changes will then cancel out in total.

In section 2 we outline the general procedure of the $\eta$-shift induced by the occurrence of a non-trivial $W_{B}$.

In section 3 we show the three-brane/five-brane correspondence for the case of a smooth fourfold.

In section 4 we lay the ground for the treatment of the (codimension one) singular case. We recall some points of the identification of the moduli spaces and the expression of the Hodge numbers of the fourfold in heterotic data; starting from there we show how this already gave the result $a_{f}=n_{3}$ in the simple case $W_{B}=0$ ([1] showed this in the smooth case by a different procedure; we build on this type of argument in section 3 ). Then we 
derive the (functional, this will be explained below) change in the index of bundle moduli under the shift in $\eta$ by $W_{B}$.

In section 5 the necessary informations about deformations of $C$ are derived. For this we investigate the corresponding question for $C$ in the base surface $B=B_{2}$ and in the elliptic surface $\mathcal{E}$ which lies in $Z$ above $C$.

In section 6 we put our informations about (functional) changes in the Hodge numbers of the fourfold together and show that they cancel each other thereby reducing the proof of our result to the original case $\left(W_{B}=0\right)$.

\section{Testing $a_{f}=n_{3}$ with $W_{B}$ turned on}

We consider the heterotic string on $\pi: Z \rightarrow B_{2}$ with a section $\sigma$ and specify a vector bundle $V=E_{8} \times V_{2}$ where we fix $V_{1}$ to be an $E_{8}$ bundle and $V_{2}=E_{8}, E_{7}, E_{6}$ or $S U(n)$.

Under the decomposition $\sigma H^{2}\left(B_{2}\right)+H^{4}\left(B_{2}\right)$, where the base cohomology is pulled back by $\pi^{*}$, the second Chern class of $V_{2}$ respectively the fundamental characteristic class of $E_{8,7,6}$ bundles, is given by

$$
\lambda\left(V_{2}\right)=\eta \sigma+\pi^{*}(\omega)
$$

where $\lambda\left(V_{2}\right)=c_{2}\left(V_{2}\right)$ for $V_{2}=S U(n)$ and $\lambda\left(V_{2}\right)=c_{2}\left(V_{2}\right) / C$ with $C=60,36,24$ for $E_{8}, E_{7}, E_{6}$ bundles. Note further that $\eta \in H^{2}\left(B_{2}\right)$ is arbitrary and $\omega \in H^{4}\left(B_{2}\right)$ is determined in terms of $\eta$. The explicit expressions for the characteristic classes of $E_{8}$ bundles and $S U(n)$ bundles are given in [1] for $E_{7}$ and $E_{6}$ bundles see [9]. A corresponding decomposition of the second Chern class of $Z$ gives

$$
c_{2}(Z)=12 c_{1} \sigma+c_{2}+11 c_{1}^{2}
$$

The general condition for anomaly cancellation with five-branes is

$$
\lambda\left(V_{1}\right)+\lambda\left(V_{2}\right)+W=c_{2}(Z)
$$

where $W$ is the cohomology class of the five-branes. As we want to treat the case that one has, besides some elliptic fibers wrapped by five-branes (the case already treated in

[1]), also a wrapped curve $C$ in the base let us now assume for the five-brane class a corresponding decomposition in a part consisting of base cohomology (to be considered embedded via $\sigma$ ) and a fiber part

$$
W=W_{B}+a_{f} F
$$


where $W$ has to be effective [6], which in the cases considered is equivalent to $W_{B}$ being effective and $a_{f} \geq 0$ [7]. Now the $\sigma H^{2}\left(B_{2}\right)$ part of (2.4) gives the condition

$$
\eta_{1}+\eta_{2}+W_{B}=12 c_{1}
$$

On the other hand one gets an expression for the number of five-branes wrapping still just the elliptic fiber of $Z$

$$
a_{f}=12+10 c_{1}^{2}-\omega_{1}-\omega_{2}
$$

This gives also a prediction for the number of three-branes on the $F$-theory side which is proportional to the Euler number of the Calabi-Yau four-fold, one has $\chi(X) / 24=n_{3}$. Now the fibration structure of the $F$-theory base $B_{3}$ is described by assuming the $\mathbf{P}^{\mathbf{1}}$ bundle over $B_{2}$ to be a projectivization of a vector bundle $Y=\mathcal{O} \oplus \mathcal{T}$, where $\mathcal{T}$ is a line bundle over $B_{2}$ and the cohomology class $t=c_{1}(\mathcal{T})$ encodes the $\mathbf{P}^{\mathbf{1}}$ fibration structure. Now the duality with the heterotic side is in the case $W_{B}=0$ implemented by choosing the $E_{8} \times V_{2}$ bundle so that the $\eta$ class of the $E_{8}$ bundle is given by $\eta_{1}=6 c_{1}\left(B_{2}\right)+t$ and the $\eta$ class of the $V_{2}$ bundle by $\eta_{2}=6 c_{1}\left(B_{2}\right)-t$.

As mentioned, we will now allow five-branes to wrap a curve $C$ of class $W_{B}$ in the base $B_{2}$. This modifies the direct relation between the $\eta$ classes $\eta_{1,2}=6 c_{1} \pm t$ and the $\mathbf{P}^{1}$ fibration described by $t$. We will describe what makes it possible to have still the relation $a_{f}=n_{3}$. In accordance with condition (2.5) we must deviate from the $\eta_{1,2}=6 c_{1} \pm t$ set-up by redefining

$$
\eta_{1} \rightarrow \eta_{1}-W_{B}
$$

Before we come to the actual computations let us note that in general the relation for the number of three-branes will be modified due to the appearance of the $F$-theory analog of $M$-theory four-flux which one is even forced to turn on if $\chi(X) / 24$ is not integral [10], [11]. One has the generalized relation

$$
\frac{\chi(X)}{24}=n_{3}+\frac{1}{2} \int_{X} G \wedge G .
$$

In particular for $S U(n)$ bundles it was expected from an argument given in [12] that $G^{2}=-\sum_{i} \frac{1}{2} \pi_{*}\left(\gamma_{i}^{2}\right)$, i.e there is a relation between four-flux and discrete twist (which appears in the $S U(n)$ bundle construction). Such a relation could be proved indirectly by an explicit computation in [9].

Since a vector bundle is determined by specifying the $\eta$ class which has to satisfy a bound [3], [4], [9], [13] let us note here that (the form of) the bound of [4] (for the new $\eta$ ) is not modified if we include a non-zero $W_{B}$ as discussed in [9]. 


\section{The smooth case}

As mentioned in the introduction, we consider here the heterotic string on $Z$ with an $E_{8} \times E_{8}$ bundle corresponding to $F$-theory on a smooth $X$. In order to perform the $\eta$-shift in the vector bundle, let us recall that the fundamental characteristic class of an $E_{8}$ bundle is

$$
\lambda(V)=\eta \sigma-15 \eta^{2}+135 \eta c_{1}-310 c_{1}^{2}
$$

and the anomaly cancellation condition then determines the number of five-branes $a_{f}$ wrapping elliptic fibers of $Z$. Now we have two choices to perform the shift either in the first or in the second $E_{8}$ factor. For the shift in $\eta_{1}$ we get

$$
a_{f}=c_{2}+91 c_{1}^{2}+30 t^{2}+15 W_{B}^{2}-45 W_{B} c_{1}-30 W_{B} t
$$

whereas for shifting $\eta_{2}$ we get

$$
a_{f}=c_{2}+91 c_{1}^{2}+30 t^{2}+15 W_{B}^{2}-45 W_{B} c_{1}+30 W_{B} t
$$

which for vanishing $W_{B}$ of course reduces to the known expression derived in [1]. Note that if we perform the shift (assuming here $t \geq 0$ ) in $\eta_{1}$ the $\eta$-bound requires $W_{B} \leq c_{1}+t$

whereas for $\eta_{2}$ the bound requires $t \leq c_{1}$. Now if we perform the shift in $\eta_{2}$ then the bound for that bundle requires $t+W_{B} \leq c_{1}$ whereas from $\eta_{1}$ comes no further condition to $t$ since $6 c_{1}+t>5 c_{1}$.

On the other hand, the number of three-branes $n_{3}=\chi(X) / 24$ to be included for a consistent $F$-theory compactification on a smooth $X$, as computed in [14], is

$$
n_{3}=12+15 \int_{B_{3}} c_{1}\left(B_{3}\right)^{3} .
$$

We will be interested now to see how the effect of wrapping a five-brane on a curve $C$ in $B_{2}$ of class $W_{B}$ on the heterotic side, reflected in a blow-up along $C$ in $B_{3}$, will change the number of three-branes on the $F$-theory side. A blow-up of $C$ in $B_{3}$ produces a three-fold $\pi: \tilde{B}_{3} \rightarrow B_{3}$ with

$$
c_{1}\left(\tilde{B}_{3}\right)=\pi^{*} c_{1}\left(B_{3}\right)-E
$$

where $E$ denotes the exceptional divisor, a ruled surface over $C$. It is useful to recall here that the well known case of a blow-up of a point on a surface leading to an exceptional $\mathbf{P}^{\mathbf{1}}$ 
of self-intersection number -1 generalizes to a relation (in $H^{4}\left(\tilde{B}_{3}\right)$ ) for the ruled surface over $C\left(E^{3}\right.$ is here a number which occurs as prefactor of $\left.l\right)$

$$
E^{2}=-\pi^{*} W_{B}-E^{3} l
$$

where $l$ denotes the fiber of the ruled surface $E$ with $E \cdot l=-1$ (for a proof of this relation cf. 90]). With this in hand we can proceed and compute the number of three-branes which are expected to match the $a_{f}$ five-branes. What we find is

$$
n_{3}=12+15 \int_{B_{3}} c_{1}\left(B_{3}\right)^{3}-3 W_{B} c_{1}\left(B_{3}\right)-E^{3} .
$$

Since $B_{3}$ is a $\mathbf{P}^{\mathbf{1}}$ bundle over $B_{2}$ obtained by projectivizing a vector bundle, as explained above, the adjunction formula gives

$$
c_{1}\left(B_{3}\right)=c_{1}+2 r+t
$$

The triple intersection of the ruled surface $E$ is given by (cf. [9])

$$
E^{3}=-\int_{W_{B}} c_{1}\left(N_{B_{3}} C\right)=-\left(c_{1}\left(B_{3}\right) W_{B}-\chi(C)\right)
$$

With $12=\int_{B_{2}} c_{1}^{2}+c_{2}$ for the rational $B_{2}$ and that $r(r+t)=0$ in the cohomology ring of $B_{3}$ (i.e. the sections of the line bundles $\mathcal{O}(1)$ and $\mathcal{O}(1) \otimes \mathcal{T}$ over $B_{2}$ cf. [1] have no common zeros) and noting that $W_{B}$ is embedded via $W_{B} r$ into $B_{3}$ so that the term $W_{B} r$ is actually $W_{B} r^{2}$, leading after integration over the $\mathbf{P}^{\mathbf{1}}$ fibers of $B_{3}$ to $-W_{B} t$, one gets with adjunction $\chi(C)=-W_{B}\left(W_{B}-c_{1}\right)$ the final expression for the number of three-branes expressed in terms of $B_{2}$ data

$$
n_{3}=\int_{B_{2}} c_{2}+91 c_{1}^{2}+30 t^{2}+15 W_{B}^{2}-45 W_{B} c_{1}+30 W_{B} t
$$

matching the $a_{f}$ heterotic five-branes in case of the shifted $\eta_{2}$ (for the $\eta_{1}$ shift one has to replace $t$ by $-t$ on the $F$-theory side). 


\section{The singular case I: comparison of moduli spaces}

We will consider here the situation where the fibers of the Calabi-Yau four-fold degenerate of type $A-D-E$ over codimension one (actually over $B_{2}$ ) in $B_{3}$ corresponding to an unbroken gauge group of the same type which on the heterotic side comes from an $E_{8} \times V_{2}$ bundle over $Z$.

The codimension one degeneration is established (cf. [9]) for the $\mathbf{A}$ series by setting $t=c_{1}$, for the $\mathbf{D}$ series only in the case $D_{4}$ a codimension one condition can be established for $t=2 c_{1}$ and for the $\mathbf{E}$ series the conditions are $t=3 c_{1}, 4 c_{1}$ for $E_{6}$ resp. $E_{7}$.

Note that we have to perform the shift always in the $E_{8}$ bundle since the choice of $t$ (for codimension one) sets the $\eta$ class on its lower bound.

Now, in the case of a codimension one degeneration of the elliptic fiber one can write down a general expression for the Euler number of the corresponding Calabi-Yau four-fold (which was first written down in [15] based there on toric computer analysis and proved in [9]) and is given by

$$
\chi(X)=288+360 \int_{B_{3}} c_{1}\left(B_{3}\right)^{3}-r(G) c(G)(c(G)+1) \int_{B_{2}} c_{1}\left(B_{2}\right)^{2}
$$

where $r(G)$ and $c(G)$ denoting the rank and the Coxeter number of the $A$-D-E gauge group $G$ respectively. If however now one would like to apply the blow-up procedure on the basis of this formula in order to get the change in the number of three-branes, this could not be done directly since the fibration structure of $B_{3}$ changes under the blow-up. Although one could refine the derivation of (4.1) in the following we will discuss (as considered in the introduction) a different approach to test $a_{f}=n_{3}$ which will provide us also with the change in the hodge numbers after the blow-up.

The shift redefinition described above for $\eta_{1}$ will change of course $a_{f}$ and so better $n_{3}$ too. But this is not the point of what we will follow, as these changes are still captured by the transport provided by the re-expression (cf. below) between the number of bundle moduli and the second Chern class of $V_{2}$. What we will follow is how this transport changes. This is a change caused just by the change in the $\sigma H^{2}\left(B_{2}\right)$ part of $c_{2}\left(V_{2}\right)$, whereas the changes which occurs because of just using 'another' $\eta_{1}$ occurs in the $H^{4}\left(B_{2}\right)$ part (whose changing influence on $a_{f}$ is still captured by the original construction).

Therefore let us first recall some facts about the general comparison of the $F$-theory and heterotic moduli spaces and spectra. These will provide us with relations expressing the Calabi-Yau four-fold hodge numbers in terms of heterotic data. We will then show 
how these relations have to be modified when on the heterotic side five-branes wrapping curves in the base are included.

The moduli in a $4 \mathrm{D} \mathrm{N}=1$ heterotic compactification on an elliptic CY, as well as in the dual $F$-theoretic compactification, break into "base" parameters which are even (under the natural involution of the elliptic curves), and "fiber" or twisting parameters; the latter include a continuous part which is odd, as well as a discrete part. In [12] all the heterotic moduli were interpreted in terms of cohomology groups of the spectral covers, and identified with the corresponding $F$-theoretic moduli in a certain stable degeneration. For this one uses the close connection of the spectral cover and the $A-D-E$ del Pezzo fibrations. For the continuous part of the twisting moduli, this amounts to an isomorphism between certain abelian varieties: the connected component of the heterotic Prym variety (a modified Jacobian) and the $F$-theoretic intermediate Jacobian. The comparison of the discrete part, involving gamma class and four-flux, refines the matching of $a_{f}$ five-branes and $n_{3}$ three-branes, as mentioned above.

By working with elliptically fibered $Z$ one can extend adiabatically the known results about moduli spaces of $G$-bundles over an elliptic curve $E=T^{2}$, of course taking into account that such a fiberwise description of the isomorphism class of a bundle leaves room for twisting along the base $B_{2}$. The latter possibility actually involves a two-fold complication: there is a continuous as well as a discrete part of these data. It is quite easy to see this for $G=S U(n)$ : in this case $V_{2}$ can be constructed via push-forward of the Poincare bundle on the spectral cover $C \times_{B} Z$, possibly twisted by a line bundle $\mathcal{N}$ over the spectral surface $C$ (an $n$-fold cover of $B_{2}$ (via $\pi$ ) lying in $Z$ ), whose first Chern class (projected to $B_{2}$ ) is known from the condition $c_{1}\left(V_{2}\right)=0$. So $\mathcal{N}$ itself is known up to the following two remaining degrees of freedom: first a class in $H^{1,1}(C)$ which projects to zero in $B$ (the discrete part), and second an element of $\operatorname{Jac}(C):=\operatorname{Pic}_{0}(C)$ (the continuous part; the moduli odd under the elliptic involution).

The continuous part is expected [1] to correspond on the $F$-theory side to the odd moduli, related there to the intermediate Jacobian $J^{3}(X)=H^{3}(X, \mathbf{R}) / H^{3}(X, \mathbf{Z})$ of dimension $h^{2,1}(X)$, so that the following picture emerges (ignoring the Kahler classes on both sides). The moduli space $\mathcal{M}$ of the bundles is fibered $\mathcal{M} \rightarrow \mathcal{Y}$, with fiber $\operatorname{Jac}(C)$. There is a corresponding picture on the $F$-theory side: the moduli space there is again fibered. The base is the moduli space of complex deformations, the fiber is (up to the discrete $G$-flux twisting parameters) the intermediate Jacobian, one has

$$
h^{2,1}(Z)+h^{1}(Z, a d V)+1=h^{3,1}(X)+h^{2,1}(X) .
$$


Here the number of bundle moduli $h^{1}(Z, a d V)=n_{e}+n_{o}$, even or odd under the involution coming from the involution on the elliptic fiber, can be computed using a fixed point theorem [1] and then first effectively computing the character-valued index $I=n_{e}-n_{o}$. So one gets

$$
h^{1}(Z, a d V)=I+2 n_{o}
$$

where the index $I$ is given by an integral over the fixed point set [1] and can be expressed in terms of the characteristic class of $V$ (cf. the last section and [16]) $(r k=r(V))$

$$
I=r k-4(\lambda(V)-\eta \sigma)+\eta c_{1} .
$$

Note that this expression applies to vector bundles which are invariant under the involution of the elliptic fiber ( $\tau$-invariant) which is the case for $E_{8}, E_{7}$ and $E_{6}$ bundles (whose characteristic classes $\lambda(V)$ were computed using the parabolic bundle construction which includes no additional twist which would break the $\tau$-invariance); also $S U(n)$ bundles where $n$ is even are $\tau$-invariant but bundles with $n$ odd (since one can twist with a line bundle in the spectral cover bundle construction introducing thereby an additional term into $\lambda(V))$ are in general not $\tau$-invariant, however, the codimension one condition on the $F$-theory side (specifying $t$ ) leads to the vanishing of the additional term in $\lambda(V)$ and in this case bundles with $n$ odd are $\tau$-invariant.

Note also that one can give [12] an interpretation of all the bundle moduli in terms of even respectively odd cohomology of the spectral surface, including an interpretation of the index as giving essentially the holomorphic Euler characteristic of the spectral surface. More precisely one can identify the number of local complex deformations $h^{2,0}(C)$ of $C$ with $n_{e}$ respectively the dimension $h^{1,0}(C)$ of $\operatorname{Jac}(C):=\operatorname{Pic}_{0}(C)$ with $n_{o}$.

In this way one gets from a spectrum comparison the following relations [2], [16] (where we assume that the elliptic fiber degenerates over codimension one in $B_{3}$ )

$$
\begin{aligned}
& h^{1,1}(X)=h^{1,1}(Z)+1+r \\
& h^{2,1}(X)=n_{o} \\
& h^{3,1}(X)=h^{2,1}(Z)+I+n_{o}+1
\end{aligned}
$$

From these relations one finds (this is recalled briefly below) that $a_{f}=n_{3}$ in the case that all five-branes wrap elliptic fibers only. For this one first expresses (from the heterotic identification) the Hodge numbers of $X$ purely in data of the common base $B_{2}$, then one uses the expression for the index $I$ (cf. [2]) and finally takes into account that on the 
$F$-theory side the $h^{2,1}(X)$ classes correspond to modes odd under the $\tau$ involution on the heterotic side.

For this recall that for an $S U(n)$ bundle one has [1] (cf. below for a discussion of the influence of the discrete twisting parameter)

$$
\begin{aligned}
c_{2}(V) & =\eta \sigma-\frac{n^{3}-n}{24} c_{1}\left(B_{2}\right)^{2}-\frac{n}{8} \eta\left(\eta-n c_{1}\left(B_{2}\right)\right) \\
& =\eta \sigma+\omega
\end{aligned}
$$

Furthermore the index $I=n_{e}-n_{o}$ is computed as [1], [12] (cf. also the appendix of [9])

$$
I=n-1+\frac{n^{3}-n}{6} c_{1}\left(B_{2}\right)^{2}+\frac{n}{2} \eta\left(\eta-n c_{1}\left(B_{2}\right)\right)+\eta c_{1}\left(B_{2}\right)
$$

so that $I=I_{1}+I_{2}$ becomes with $r k=r k_{1}+r k_{2}$, the sum of the ranks of the two vector bundles (so that $r=8+8-r k$ is the rank of the unbroken gauge group)

$$
I=r k-4\left(\omega_{1}+\omega_{2}\right)+\left(\eta_{1}+\eta_{2}\right) c_{1}
$$

leading with (2.5) to the result indicated above. Note that the index for $S U(n)$ bundles was computed in [1] and for $E_{8}$ bundles in [16].

Before we proceed to the refinements in the situation $W_{B} \neq 0$ let us briefly recall how one proceeds from in the argument for $n_{3}=a_{f}$ in the case $W_{B}=0$ [2]. From the last expression for the total index one gets

$$
\begin{aligned}
I & =r k-4\left(c_{2}\left(V_{1}\right)+c_{2}\left(V_{2}\right)\right)+48 c_{1}\left(B_{2}\right) \sigma+12 c_{1}^{2}\left(B_{2}\right) \\
& =r k-4\left(12 c_{1}\left(B_{2}\right) \sigma+c_{2}\left(B_{2}\right)+11 c_{1}^{2}\left(B_{2}\right)\right)+4 a_{f}+48 c_{1}\left(B_{2}\right) \sigma+12 c_{1}^{2}\left(B_{2}\right) \\
& =r k-\left(48+28 c_{1}^{2}\left(B_{2}\right)\right)+4 a_{f}
\end{aligned}
$$

From $\chi(X) / 6-8=h^{1,1}(X)-h^{2,1}(X)+h^{3,1}(X)$ (cf. [14]), i.e.

$$
n_{3}=2+\frac{1}{4}\left(h^{1,1}(X)-h^{2,1}(X)+h^{3,1}(X)\right)
$$

and the relations

$$
\begin{aligned}
& h^{1,1}(X)=12-c_{1}^{2}\left(B_{2}\right)+r \\
& h^{3,1}(X)=12+29 c_{1}^{2}\left(B_{2}\right)+I+n_{o}
\end{aligned}
$$


which are refinements of (4.5) using $3 h^{1,1}(Z)=h^{1,1}\left(B_{2}\right)+1=c_{2}\left(B_{2}\right)-1=11-c_{1}\left(B_{2}\right)^{2}$ and $\chi(Z)=-60 c_{1}^{2}\left(B_{2}\right)$ (cf. [17]) then one computes indeed

$$
n_{3}=2+\frac{1}{4}\left(12-c_{1}^{2}\left(B_{2}\right)+16-r k+12+29 c_{1}^{2}\left(B_{2}\right)+I\right)=a_{f}
$$

So far the original argument. Now, in the case $W_{B} \neq 0$ observe that after the redefinition shift has been made, the index $I=I_{1}+I_{2}$ can be rewritten as

$$
I=I_{\text {old }}-W_{B} c_{1}=r k-4\left(\omega_{1}+\omega_{2}\right)+12 c_{1}^{2}-W_{B} c_{1}
$$

which is the usual index (of course depending now on the new $\eta$ ) shifted by $\Delta I=-W_{B} c_{1}$.

In the general case one has besides the geometric and bundle moduli given above also to take into account the possible deformations $\operatorname{de}_{Z} C$ of the actually chosen curve $C$ (which is wrapped by the five-brane) inside the cohomology class $W_{B}=[C]$ which we are going to describe now.

\section{The singular case II: deformations of $C$}

We will treat first the deformations of $C$ in $B$ (always considered to be embedded via $\sigma$ in $Z$ ) and in the elliptic surface $\mathcal{E}$ lying above $C$.

Note that in the following we will in the case of possible doubt where, like for $C$, a self-intersection is to be taken, denote the self-intersection of $b$ considered as a curve in the surface $\mathcal{E}$ by $C_{\mathcal{E}}^{2}$ to distinguish it from the self-intersection $C_{B_{2}}^{2}$ in $B=B_{2}$

3 we assume that there is only one cohomologically independent section $\sigma$ of the elliptic fibration $\pi: Z \rightarrow B$

4 Note that in principle one has in $\omega=\omega_{\gamma=0}-\frac{1}{2} \pi_{*} \gamma^{2}$ a $\gamma$ related term which does, in contrast to $-4 \omega$, not occur in the expression for the index (4.7) (here $\gamma$ is the discrete twisting parameter, cf. [1], [9], [12], which in some cases even has to be present); therefore this $\gamma$ related term will also appear besides $a_{f}$ in (4.9); nevertheless we have suppressed this term, for we will assume, as motivated in [12] and indirectly proved for the codimension one singular case in [9], that it corresponds with a four-flux term on the $F$-theory side, so that by (2.8) the sought for direct relation between $a_{f}$ and $n_{3}$ is still maintained. 


\subsection{The deformations of $C$ in $B$}

We have a 'local' information $h^{0}\left(C, N_{B} C\right)$ about deformations of $C$ in $B$ as well as a 'global' one $\operatorname{def}_{B_{2}}(C)=h^{0}\left(B_{2}, \mathcal{O}(C)\right)-1$. Using Riemann-Roch

$$
\sum_{i=0}^{2}(-1)^{i} h^{i}\left(B_{2}, \mathcal{O}(C)\right)=h^{0}\left(C, N_{B_{2}} C\right)-h^{1}\left(C, N_{B_{2}} C\right)+\chi\left(B_{2}, \mathcal{O}\right)
$$

we get (with $\chi\left(B_{2}, \mathcal{O}\right)=1$ from Noether for our rational $B_{2}$ )

$$
\operatorname{def}_{B}(C)=h^{0}\left(C, N_{B_{2}} C\right)-h^{1}\left(C, N_{B} C\right)+s-h^{2}(B, \mathcal{O}(C))
$$

with the local terms

$$
h^{0}\left(C, N_{B} C\right)-h^{1}\left(C, N_{B} C\right)=\frac{1}{2} \chi(C)+\operatorname{deg} N_{B} C=\frac{C c_{1}+C^{2}}{2}
$$

Let us investigate the two higher cohomological corrections. First for any curve $C$ on a rational surface $B$

$$
h^{2}\left(\mathcal{O}_{B}(C)\right)=h^{0}(\mathcal{O}(K-C))=0
$$

which can be seen from the exact sequence

$$
0 \rightarrow \mathcal{O}_{B} \rightarrow \mathcal{O}_{B}(C) \rightarrow \mathcal{O}_{C}(C) \rightarrow 0
$$

whose associated long exact cohomology sequence reads

$$
\begin{aligned}
0 & \rightarrow h^{0}\left(B, \mathcal{O}_{B}\right) \rightarrow h^{0}\left(B, \mathcal{O}_{B}(C)\right) \rightarrow h^{0}\left(\mathcal{O}_{C}(C)\right) \\
& \rightarrow h^{1}\left(B, \mathcal{O}_{B}\right) \rightarrow h^{1}\left(B, \mathcal{O}_{B}(C)\right) \rightarrow h^{1}\left(\mathcal{O}_{C}(C)\right) \\
& \rightarrow h^{2}\left(B, \mathcal{O}_{B}\right) \rightarrow h^{2}\left(B, \mathcal{O}_{B}(C)\right) \rightarrow h^{2}\left(\mathcal{O}_{C}(C)\right) \rightarrow \cdots
\end{aligned}
$$

Here vanishing of the last term shown and $p_{g}(B)=0$ show together that $h^{2}\left(\mathcal{O}_{B}(C)\right)=0$.

Now we have to consider the superabundance $s=h^{1}(B, \mathcal{O}(C))$ for the cases of $B=$ $B_{2}$ being a del Pezzo surface $d P_{k}\left(\mathbf{P}^{2}\right.$ blown-up in $k$ points $)$ or a Hirzebruch surface $F_{n}\left(\right.$ a $\mathbf{P}^{1}$ bundle over $\left.\mathbf{P}^{1}\right)$. The long exact sequence shows with $q(B)=p_{g}(B)=0$ that $h^{1}\left(B, \mathcal{O}_{B}(C)\right)=h^{1}\left(\mathcal{O}_{C}(C)\right)=h^{0}\left(C, K_{C}-N_{C / B}\right)$ which vanishes certainly for $0>$ $\operatorname{deg}\left(K_{C}-N_{C / B}\right)=C\left(K_{B}+C\right)-C^{2}=C K$ which is guaranteed if we assume $-K$ ample; thus for $B_{2}=F_{n}$ with $n=0,1$ and $d P_{k}$ with $k \neq 9$ the superabundance vanishes. In general we have the important consequence

$$
C \cdot c_{1}\left(B_{2}\right)>0 \Rightarrow s=0
$$


so that we find under this assumption

$$
C \cdot c_{1}\left(B_{2}\right)>0 \Rightarrow \operatorname{def}_{B}(C)=h^{0}\left(C, N_{B_{2}} C\right)=\frac{C c_{1}+C^{2}}{2} .
$$

Let us now discuss the case $B_{2}=F_{2}$. Recall that for $F_{n}$ the Kaehler cone (the very ample classes) equals the positive (ample) classes and is given 18 by the numerically effective classes $x b+y f=(x, y)$ with $x>0, y>n x$ where $b=b_{-}$is the section with $b^{2}=-n$ and $f$ the fiber. Furthermore note that an irreducible non-singular curve exists in a class $x b+y f$ exactly if the class lies in the mentioned cone or is the element $b=(1,0)$ of negative selfintersection or one of the elements $f=(0,1)$ or $k b_{+}$(with $k>0$ and $b_{+}=b_{-}+n f=(1, n)$ of $\left.b_{+}^{2}=+n\right)$ on the boundary of the mentioned cone; all of these classes together with their positive linear combinations span the effective cone $(x, y \geq 0)$. Note that $c_{1}=2 b+(n+2) f$ is positive only for $F_{0}$ and $F_{1}$ whereas for $F_{2}$ it lies only on the boundary of the positive cone.

For $F_{2}$ the Kodaira vanishing theorem tells us that $h^{1}(B, \mathcal{O}(C))=h^{1}(B, \mathcal{O}(K-C))=$ 0 if $-K+C=(x+2, y+4)$ is ample, i.e. for $y>2 x, x>-2$; so clearly the superabundance will vanish for all ample $C=(x, y)$ (where even $x>0$ ) and for $f$. Note that for $f$ we see directly that $\operatorname{def}_{F_{n}} f=1=e(f) / 2+f^{2}$ (the expression from (5.3) ) and similarly for $b$ with $n \neq 0$ that $\operatorname{def}_{F_{n}} b=0$ and $e(b) / 2+b^{2}=1-n \leq 0$; so for the case $B=F_{2}$ the formal expression which should give the number of actual deformations has to be modified, and - as this case is in a further respect somewhat exceptional as we will see in the next subsection - we will exclude it. Finally for $k b_{+}$one sees that $k b_{+} \cdot c_{1}\left(F_{2}\right)=4 k>0$ making, according to the argument given above, $s$ again vanish in this case although $c_{1}\left(F_{2}\right)$ is not numerically effective in general.

\subsection{The deformations of $C$ in the elliptic surface $\mathcal{E}$}

From the Kodaira formula for the canonical bundle of $\mathcal{E}$ one has an expression for $K_{\mathcal{E}}$ as a pull-back class (of course also $F=\pi_{\mathcal{E}}^{*} p$ with $p \in C$ )

$$
K_{\mathcal{E}}=\pi_{\mathcal{E}}^{*} K_{C}+\chi\left(\mathcal{E}, \mathcal{O}_{\mathcal{E}}\right) F
$$

Then $\chi\left(\mathcal{E}, \mathcal{O}_{\mathcal{E}}\right)$ is via Noether evaluated as $\frac{1}{12} e(\mathcal{E})$ as $c_{1}(\mathcal{E})$ has, being a certain number of fibers, vanishing self-intersection. But $e(\mathcal{E})=c_{1}\left(B_{2}\right) \cdot C$ as the elliptic fibration of the Calabi-Yau has the discriminant $12 c_{1}\left(B_{2}\right)$ so that

$$
\chi\left(\mathcal{E}, \mathcal{O}_{\mathcal{E}}\right)=c_{1}\left(B_{2}\right) \cdot C
$$


Alternatively one can see from adjunction

$$
c(\mathcal{E})=c(C) \frac{(1+r)\left(1+r+2 c_{1}\left(B_{2}\right)\right)\left(1+r+3 c_{1}\left(B_{2}\right)\right)}{1+3 r+6 c_{1}\left(B_{2}\right)}
$$

that

$$
\begin{aligned}
c_{1}(\mathcal{E}) & =\left(e(C)-c_{1}\left(B_{2}\right) \cdot C\right) F \\
e(\mathcal{E}) & =12 c_{1}\left(B_{2}\right) \cdot C
\end{aligned}
$$

Note that the number $d:=c_{1}(B) \cdot C$ has the following important interpretation: from (5.9), (5.10) one has $C_{\mathcal{E}} \cdot c_{1}(\mathcal{E})=e_{C}-c_{1}(B) \cdot C_{B}\left(\right.$ as $c_{1}(\mathcal{E})$ is a pull-back class, i.e. a number of fibers) so that with adjunction $-e_{C}=C_{\mathcal{E}}^{2}-C_{\mathcal{E}} \cdot c_{1}(\mathcal{E})$ inside $\mathcal{E}$ one gets

$$
C_{\mathcal{E}}^{2}=-c_{1}(B) \cdot C_{B}
$$

This gives after (5.7) another important criterion

$$
C \cdot c_{1}\left(B_{2}\right)>0 \Rightarrow d e f_{\mathcal{E}} C_{\mathcal{E}}=0
$$

So except for the case $B=F_{2}, C=b=b_{-}$we have no further deformations in the vertical direction; in the mentioned exceptional case one had $\mathcal{E}=b \times F$ showing the obvious deformation. Note that in this case we had from the expression (5.3) that $e(b) / 2+b^{2}=$ $1-2=-1$. As in this exceptional case one has one deformation in $Z$ and no deformation in the base $B$ which the $F$-theory side could see directly we will exclude it from our final arguments.

\section{Examples}

To give some examples note that the first three cases $d=0,1,2$ leading to $e(\mathcal{E})=$ $0,12,24$ correspond to $b \times F, d P_{9}, K 3$. They occur in well-known circumstances if we choose $B_{2}=F_{n}$ with $n=2,1,0$. In that case occurs the mentioned $\mathcal{E}$ over the base $C=b=b_{-}$of self-intersection $-n$ of the Hirzebruch surface: the first case gives actually one of the two exceptional divisors (the other is the base $F_{2}$ itself) of the STU CalabiYau $\mathbf{P}_{1,1,2,8,12}(24)$ which consists in a ruled surface over the elliptic fiber (this is the just another way to consider the product $b \times F$ fibered) [19], [15]; the second case gives the $d P_{9}$ studied for example in [20], which occurs not just in this set-up but over any exceptional curve $\left(b^{2}=-1, b \cdot c_{1}\left(B_{2}\right)=1\right.$; rationality and the second property imply the first) in a del Pezzo base; finally for $F_{0}$ one gets the well-known $K 3$ fibers, which occur of course more generally also over each fiber of any Hirzebruch base (adjunction shows $d=2$ ). 


\subsection{The deformations of $C$ in $Z$}

The total deformation space can be considered fibered together out of the pieces investigated so far (cf. [8]).

Concerning $\operatorname{def}_{Z} C$ consider

$$
0 \rightarrow N_{C / B} \rightarrow N_{C / Z} \rightarrow N_{B / Z} \otimes \mathcal{O}_{C} \rightarrow 0
$$

the last term being again $N_{C / \mathcal{E}}$. To show that $H^{0}\left(N_{B / Z} \otimes \mathcal{O}_{C}\right)=0$ in order that $d e f_{C} B=$ $d e f_{C} Z$ corresponds exactly with the already formulated condition that there are no further deformations of $C$ in $\mathcal{E}$ if $C$ has there (!) negative self-intersection: for $\operatorname{deg} N_{C / \mathcal{E}}=C_{\mathcal{E}}^{2}<0$ and $H^{0}$ vanishes just as in the argument for $s=0$ given above.

\section{A remark about skew curves}

Although we will not treat this most general case (where one would have to 'connect' blow-up's and three-branes on the $F$-theory side) let us at least point to the possibility that the total physical moduli space will include even deformations of $C+a_{f} F$ away from the reducibility into base (horizontal) and fiber (vertical) curves, leading to curves lying 'skew' to the elliptic fibration.

Until yet we have considered two possible cases where a heterotic five-brane wraps a curve either of class $a_{f} F$, i.e. of the purely vertical type - a reducible sum of $a_{f}$ fibers, or $W_{B}$, the purely horizontal type wrapping a base curve $C$. Of course that choice of a base curve realizing the cohomology class $W_{B}$ involves already considerable freedom in itself; to give an example, for $B_{2}=\mathbf{P}^{2}$ and $W_{B}=2 L$ one can choose either a smooth conic, giving a rational curve $C$ of multiplicity 1 , or two crossing lines or even two times the same line, giving a rational curve $C$ of multiplicity 2 (cf. also [5]). What concerns the distribution of the vertical class $a_{f} F$ in a set of curves realizing it, one has considerable lesser freedom as one can only make the in general disjoint $a_{f}$ fibers coinciding in some subsets corresponding to a decomposition of the number $a_{f}$. So the set-up up to now would lead one in general to consider the wrapping of the reducible curve $C+a_{f} F$. Of course this realizes only a sublocus of very degenerate possibilities for a curve of cohomology class $C+a_{f} F$ as it may be actually possible to find an irreducible curve (of this cohomology class) inside $Z$, or more precisely, as we can assume, actually in the elliptic surface $\mathcal{E}$ over $C$ (cf. also [8]).

This is by no means trivial as for example in the case that the base curve $C$ is rational and that $h^{2,0}(\mathcal{E})=0$ resp. 1, i.e. $\mathcal{E}$ is either a rational elliptic $\left(d P_{9}\right)$ surface or a $K 3$. 
In that case the class $D:=C+a_{f} F$ is not representable by an irreducible smooth curve because, although it is a section of the elliptic fibration of $\mathcal{E}$ and so rational, adjunction shows that $-2=D_{\mathcal{E}}^{2}-D \cdot c_{1}(\mathcal{E})$; but in the $d P_{9}$ case $c_{1}(\mathcal{E})=F$ and so the right hand side is $-1+2 a_{f}-1$, i.e. $a_{f}=0$, whereas for $K 3$ it is just $-2+2 a_{f}$ showing again $a_{f}=0$. Here we used the fact that $C_{\mathcal{E}}^{2}=-1,2$ on $d P_{9}, K 3$ as one sees again from adjunction.

Note as a final remark that one has quite a remarkable curve counting. On $d P_{9}$ one has for the number $n_{1, \beta}$ of rational curves in the class $C+\beta F$, as counted by the GromovWitten invariants and evaluated by mirror symmetry [20], $\left(q=e^{2 \pi i \tau} ; \theta_{E_{8}}(\tau)\right.$, the theta function of the $E_{8}$ lattice being given by $\frac{1}{2} \sum_{k=\text { even }} \theta_{k}^{8}(\tau)=E_{4}(\tau)$ with the Jacobi theta functions $\theta_{k}$ and the Eisenstein series of weight $4 ; \eta$ is the Dedekind eta-function)

$$
\sum_{\beta} n_{1, \beta} q^{\beta}=q^{\frac{1}{2}} \frac{\theta_{E_{8}}(\tau)}{\eta^{12}(\tau)}
$$

Corresponding results for $\alpha C+\beta F$ with $\alpha>1$ are given in [21]. Similarly in the $K 3$ fiber $\mathbf{P}_{1,1,4,6}(12)$ (of the $S T U$ Calabi-Yau of degree 24) occurring over fibers of $F_{2}$ one has

$$
n_{\alpha, \beta}=c_{S T U}(\alpha \beta)
$$

where

$$
\sum_{n \geq-1} c_{S T U}(n) q^{n}=\frac{E_{4} E_{6}}{\eta^{24}}(\tau)
$$

Note that these invariants can be really number of curves or more generally Euler numbers of the deformation space of (or of the moduli space of flat $U(1)$ bundles over) the curve (in general one has to consider a certain obstruction bundle). In particular they don't tell us whether there is really an irreducible member besides that curve realization of the class $\alpha C+\beta F$ which is just given by the reducible curve consisting (for $\alpha=1$, say) in the base curve $C$ and a number of $\beta$ fibers. Further they don't tell us whether really a correspondingly big deformation space is realized in the elliptic surface $\mathcal{E}$, let alone in the Calabi-Yau three-fold; even if an irreducible skew realization exists this does not necessarily mean that this realization is connected (in the sense of going smoothly through supersymmetric realizations) to the reducible realization. 


\section{The singular case III: computation}

By now we are prepared with the necessary background, in order to handle the $\eta$-shift and see how the relations for the Hodge numbers of the Calabi-Yau four-fold in terms of the heterotic data will change. To keep things as clear as possible we will separate the changes on the heterotic side from those appearing in the Calabi-Yau four-fold.

\section{Heterotic Side}

On the route of our second strategy we found up to now two important things: the shift in the index and the expression for the deformation (the unspecified $c_{1}$ refers to $B$ )

$$
\begin{gathered}
\Delta I=-C c_{1} \\
\operatorname{def}_{Z} C=\frac{C^{2}+C c_{1}}{2}
\end{gathered}
$$

(Concerning the first relation note that this is the change in the functional form of $I$ as a function of $\eta_{1}$, i.e. for the argument to be given we are not interested in the change coming from just applying the usual index expression to the new, shifted $\eta_{1}$. Concerning the second relation note that, as indicated earlier, we exclude the exceptional case $B=F_{2}, C=b_{-}$.)

\section{F-Theory Side}

On the F-theory side the heterotic $\eta$-shift is understood as blowing up of the corresponding curve $C$ in the base of the Calabi-Yau four-fold which leads to a new divisor and so to a new Kaehler class, thus

$$
h^{1,1}(X) \rightarrow h^{1,1}(X)+1
$$

also, this blow up leads to

$$
g_{C}=\frac{C^{2}-C \cdot c_{1}}{2}+1
$$

new 3 -cycles in $h^{2,1}\left(B_{3}\right)$ and also in $h^{2,1}(X)$, so

$$
h^{2,1}(X) \rightarrow h^{2,1}(X)+g_{C} .
$$

Finally the change in $h^{3,1}(X)$ accounts for both effects, the index-shift $\Delta I=-C c_{1}$ (as the index of the bundle moduli maps to the deformations of the fourfold, cf. (4.5) ) and the occurrence of the $C$-deformations (which become geometrical on the $F$-theory side)

$$
h^{3,1}(X) \rightarrow h^{3,1}(X)-C c_{1}+\operatorname{def} f_{B} C
$$


From the relation (4.10) for the number of three-branes we learn that the three changes in the Hodge numbers cancel out since (cf. (5.3))

$$
1-g_{C}-C c_{1}+\operatorname{def}_{B} C=0
$$

Thus, by expressing everything in data of the common base $B_{2}$, just as it was done in [2], we find that the desired matching $n_{3}=a_{f}$ of the number of heterotic five-branes wrapping elliptic fibers with the $F$-theory three-branes still holds in the more general situation with a non-trivial $W_{B}$, as the $\eta$-shift reduces this completely to the old argument in the simpler situation of $W_{B}=0$.

Let us finally make a remark on the exceptional case. For this let us compute the prediction for $h^{3,1}(X)$ from the heterotic side in case we have an $E_{8} \times E_{8}$ bundle leaving no unbroken gauge group (and so having a smooth $X$ on the $F$ side) for the case of $B_{2}=F_{2}$ and wrapping the five-brane on the base $b$ of $F_{2}$. Let us assume that $t=0$ which means we have $B_{3}=\mathbf{P}^{1} \times F_{2}$ on the $F$-side. Since $t=0$ we can either perform the $W_{B}$ shift in the first $E_{8}$ or in the second one. The index computation gives for the unshifted $E_{8}$ bundle $I=1336$ and for the shifted bundle 1216 therefore one has $h^{3,1}(X)=h^{2,1}(Z)+I+1+\operatorname{def}_{B} C=243+1336+1216+1-1=2795$. Now an independent computation (using toric geometry) of $h^{3,1}(X)$ [22] matches precisely the heterotic prediction.

Note that we have used here the 'formal' expression for $\operatorname{def}_{B} C$ which gives -1 (so to speak an 'obstruction') whereas the actual number of deformations of $b$ is 0 ; further in $Z$ the number is even +1 . This shows that this last heterotic deformation in $\mathcal{E}$ is, as one expects, not directly visible on the $F$-theory side and that on the other hand, concerning the deformations in the common visible $B=B_{2}$, the $F$-theory side 'sees' the 'obstruction' too, as the -1 is reflected there.

We would like to thank A. Klemm, D. Morrison and J. Wahl for discussions. 


\section{References}

[1] R. Friedman, J. Morgan and E. Witten, "Vector Bundles and F- Theory," Commun. Math. Phys. 187 (1997) 679, hep-th/9701162.

[2] B. Andreas and G. Curio, "Three-Branes and Five-Branes in N=1 Dual String Pairs," Phys. Lett. B417 (1998) 41, hep-th/9706093.

[3] P. Berglund and P. Mayr,"Heterotic/F-Theory Dulality from Mirror Symmetry", Adv.Theor.Math.Phys.2 (1999) 1307, hep-th/9811217.

[4] G. Rajesh, "Toric Geometry and F-theory/Heterotic Duality in Four Dimensions", JHEP 12 (1998) 18, hep-th/9811240.

[5] D.-E. Diaconescu and G. Rajesh, "Geometrical Aspects of Fivebranes in Heterotic/FTheory Duality in Four Dimensions", hep-th/9903104.

[6] R. Donagi, A. Lukas, B. A. Ovrut and D. Waldram, "Non-Perturbative Vacua and Particle Physics in M-Theory", JHEP 9905 (1999) 018, hep-th/9811168.

[7] R. Donagi, A. Lukas, B. A. Ovrut and D. Waldram, "Holomorphic Vector Bundles and Non-Perturbative Vacua in M-Theory", hep-th/9901009.

[8] R. Donagi, B. A. Ovrut and D. Waldram, "Moduli Spaces of Fivebranes on Elliptic Calabi-Yau Threefolds", hep-th/9904054.

[9] B. Andreas and G. Curio, "On Discrete Twist and Four Flux in N=1 Heterotic/FTheory Compactifications", hep-th/9908193.

[10] E. Witten, "On Flux Quantization in M-Theory and the Effective Action," J. Geom. Phys. 22 (1997) 1, hep-th/9609122.

[11] K. Dasgupta and S. Mukhi, "A Note on Low Dimensional String Compactifications," Phys. Lett. B398 (1997) 285, hep-th/9612188.

[12] G. Curio and R. Donagi, "Moduli in N=1 Heterotic/F-Theory Duality," Nucl.Phys. B518 (1998) 603, hep-th/9801057.

[13] P. Berglund and P. Mayr, "Stability of Vector Bundles from F-Theory", hepth/9904114.

[14] S. Sethi, C. Vafa and E. Witten, "Constraints on Low Dimensional String Compactifications," Nucl. Phys. B480 (1996) 213, hep-th/9606122.

[15] S. Hosono, A. Klemm, S. Theisen and S.-T. Yau, "Mirror Symmetry, Mirror Map and Applications to Complete Intersection Calabi-Yau Spaces", Nucl. Phys. B433 (1995) 501, hep-th/9406055.

[16] B. Andreas, G. Curio and D. Lüst, "N=1 Dual String Pairs and their Massless Spectra", Nucl. Phys. B507 (1997) 175, hep-th/9705174.

[17] A. Klemm, B. Lian, S.-S. Roan and S.-T. Yau, "Calabi-Yau fourfolds for M and Ftheory compactifications", Nucl. Phys. B 518 (1998) 515, hep-th/9701023.

[18] R. Hartshorne, "Algebraic Geometry", Springer 1977. 
[19] S. Kachru and C. Vafa, "Exact Results for $\mathrm{N}=2$ Compactifications of Heterotic Strings", Nucl. Phys. B450 (1995) 69, hep-th/9505105.

[20] S. Katz, P. Mayr and C. Vafa, "Mirror symmetry and exact solution of 4D N=2 Gauge theories I", hep-th/9706110.

[21] J.A. Minahan, D. Nemeschansky, C. Vafa and N.P. Warner, "E-Strings and N=4 Topological Yang-Mills Theories" Nucl. Phys. B527 (1998) 581, hep-th/9802168.

$[22]$ A. Klemm, private communication. 